\title{
Computational Fluid Dynamic Analysis of the External Rotor Supporting the Design of a Tidal Kinetic Turbine Prototype
}

\author{
Silvio Barbarelli*1, Teresa Castiglione ${ }^{2}$, Gaetano Florio ${ }^{3}$, Nino M. Scornaienchi ${ }^{4}$, \\ Giacomo Lo Zupone 5 \\ ${ }^{1}$ Department of Mechanical Energy and Management Engineering, University of Calabria, \\ Via Pietro Bucci, Rende, Italy \\ e-mail: silvio.barbarelli@unical.it \\ ${ }^{2}$ Department of Mechanical Energy and Management Engineering, University of Calabria, \\ Via Pietro Bucci, Rende, Italy \\ e-mail: teresa.castiglione@gmail.com \\ ${ }^{3}$ Department of Mechanical Energy and Management Engineering, University of Calabria, \\ Via Pietro Bucci, Rende, Italy \\ e-mail: gaetano.florio@unical.it \\ ${ }^{4}$ Department of Mechanical Energy and Management Engineering, University of Calabria, \\ Via Pietro Bucci, Rende, Italy \\ e-mail: nino_michele.scornaienchi@unical.it \\ ${ }^{5}$ Sintenergy srl, Via Reggio Modena, 50 L, Reggio Calabria, Italy \\ e-mail: glozupone@gmail.com
}

\begin{abstract}
Cite as: Barbarelli, S., Castiglione, T., Florio, G., Scornaienchi, N. M., Lo Zupone, G., Computational Fluid Dynamic Analysis of the External Rotor Supporting the Design of a Tidal Kinetic Turbine Prototype, J. sustain. dev. energy water environ. syst., 5(3), pp 332-344, 2017, DOI: http://dx.doi.org/10.13044/j.sdewes.d5.0150
\end{abstract}

\begin{abstract}
The purpose of this paper is to ascertain the reliability of a zero-dimensional approach, aimed to design a double rotor kinetic turbine prototype, by means of computational fluid dynamic analysis. The interaction between the flow and the blade of the turbine prototype external rotor is evaluated. The calculation is carried out by a three-dimensional analysis using the commercial code FLUENT 15.0. In the present work, the Reynolds Averaged Navier-Stokes approach is used, with the two-equation Realizable k-epsilon turbulence model. The blades profile is a NACA 4412 interacting with flow at an attack angle of $4^{\circ}$, which is kept constant along the blade height. The estimation of the forces acting on the blades allows a more accurate evaluation of the blade lift coefficient, which is useful to design the blades geometry and to compute the power coefficient, previously estimated by the traditional mono-dimensional approach.
\end{abstract}

\section{KEYWORDS}

Tidal currents, Turbine prototype, Zero dimensional sizing, CFD analysis of external rotor, Power coefficient evaluation, Blades lift evaluation.

\section{INTRODUCTION}

Despite actual research lines concentrating on local resources by favouring the approach of smart systems [1], marine current energy is one of the emerging renewable energy forms [2], as it constitutes a huge source of kinetic energy owing to regular tidal

\footnotetext{
* Corresponding author
} 
cycles influenced by the moon's phases [3]. Wind, wave and solar power often require backup from traditional forms of power generation [4]. On the contrary, the inherent predictability of tidal power is highly attractive for grid management, as it does not require fossil fuel backup systems. Tidal turbines are usually installed on the seabed, at prescribed locations characterized by high tidal current velocities or strong continuous ocean currents, which extract energy from the flowing water [5]. To date, few commercial-scale devices have been manufactured and tested, although many innovative tidal and ocean stream energy devices have been proposed [6]. The machines, which currently work in tidal currents, have a non-competitive Levelized Cost Of Energy (LCOE) [7].

An important contribution to the development of such technologies is given by metocean stations, mounted on monopiles or buoys equipped with meteorological sensors, which help the designer to know numerous model parameters [8].

The team Unical Sintenergy has developed a new turbine working like a kite [9]. It can be moored to the shore by a simple rope and is able to reach an equilibrium position in the sea [10]. The machine is able to work even when the tide reverses its direction [11].

After several design attempts [12], a zero-dimensional model useful for the optimal sizing of the machine is chosen. The machine has two counter rotating rotors, an external and an internal one [13]. The aforementioned model requires, however, an accurate knowledge of the fluid dynamic behaviour of the external rotor. From a methodological point of view, therefore, the present work couples a zero-dimensional approach and a CFD analysis. The scope of the CFD analysis, in particular, is the evaluation of the effects of the three-dimensional flow field on the rotor's performance. By a comparison between both approaches, it is possible to ascertain the reliability of the zero-dimensional approach in the turbine design process; in such a case, the advantages in terms of computational effort with respect to the CFD are significant.

In this paper, the sizing of a prototype with $1 \mathrm{~m}$ external diameter rotor is carried out by the application of the proposed methodology.

\section{SINTENERGY TURBINE}

Sintenergy introduced an innovative way to convert energy from tidal currents, which is efficient and cost effective (see Figure 1).

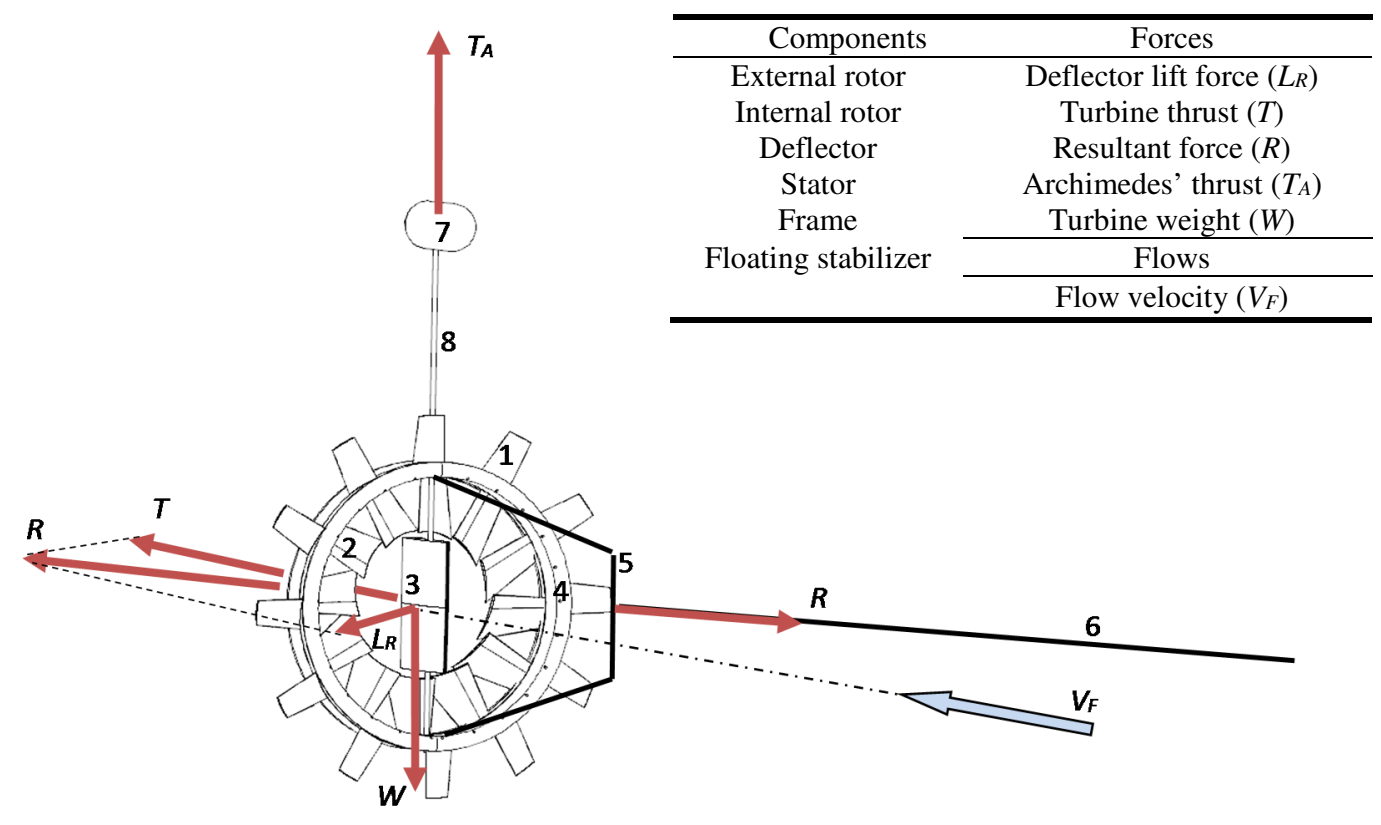

Figure 1. Working scheme of the turbine prototype 
The working principle is widely described in previous works $[14,15]$ and the most meaningful features in terms of mechanical and fluid dynamic solutions are briefly summarized.

The machine has two contra-rotating rotors $(1,2)$ turning in a stator (4), which converts the kinetic energy thanks to a built-in generator (permanent magnets). A central deflector (3) maintains the machine always facing the flow, meanwhile a vertical stabilizer, a sort of buoy (7), keeps the machine at the expected depth. The turbine is connected to the coast by means of a rope (6) stretched by the resultant $R$ (see Figure 1), which creates an angle $\beta$ with the coast. This angle does not change during the machine operation [11].

The equilibrium equations of the moments, due to the various forces, are described in [10], together with the machine design procedure. It shows that the deflector lift and drag coefficients and, particularly, the external rotor design are key elements for the whole machine design.

This last aspect is the focus of the present work. In the next sections, the sizing of the machine by means of the zero-dimensional approach is firstly illustrated, and then a three-dimensional analysis of the external rotor is carried out.

\section{SIZING OF THE PROTOYPE}

The hydrodynamic design of a generic tidal turbine provides the blade external shape, i.e. the chord and twist distributions along the blade, which result in optimal performance of the tidal turbine over its lifetime. The next figure (Figure 2) highlights the required input.

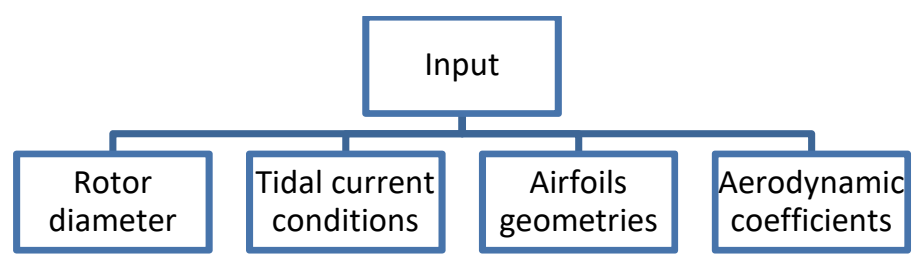

Figure 2. Input of the blade sizing

The traditionally used formulas [16] are reported in the following:

$$
\begin{gathered}
c=\frac{1}{z} \cdot \frac{\pi D_{e}}{\lambda C_{L b}} \cdot \frac{8 / 9}{\sqrt{\frac{4}{9}+\lambda^{2} \mu^{2}\left[1+\frac{2}{9 \lambda^{2} \mu^{2}}\right]^{2}}} \\
\beta_{p}=\phi-\alpha_{p} \\
\phi=\operatorname{arctg}\left(\frac{2 / 3}{\lambda \mu\left(1+\frac{2}{9 \lambda^{2} \mu^{2}}\right)}\right) \\
\mu=\frac{2 r}{D_{e}}
\end{gathered}
$$

where the various parameters are indicated in Table 1. 
Table 1. Parameters of the blade sizing

\begin{tabular}{cc}
\hline Parameter & Symbol \\
\hline Chord & $c$ \\
Blades lift coefficient & $C_{L b}$ \\
Number of blades & $z$ \\
Incidence angle & $\alpha_{p}$ \\
Tip speed ratio & $\lambda$ \\
Radius/tip diameter & $\mu$ \\
Functional angle & $\phi$ \\
Attack angle & $\beta_{p}$ \\
\hline
\end{tabular}

The Sintenergy turbine is sized by using the previous formulas and by taking into account that the ratios between the three diameters (external, mean, and internal - see Figure 1) are optimized by considering the equilibrium law of all the involved forces and the equilibrium to the rotation of the two rotors [12]. The necessity to have two rotors balanced with equal torque but rotating in opposite direction implies equal power for each rotor.

The sizing of the blades starts from the choice of the parameters $\lambda$ and $z$ of the external rotor. In a previous work [13] it was estimated that the optimal configuration is $\lambda=2.5$ and $z=6$.

The overall power can be calculated as:

$$
P=\eta_{e} \eta_{m} \rho \frac{v_{0}^{3}}{2}\left(C_{p 1} A_{1}+C_{p 2} A_{2}\right)
$$

Based on the Prandtl theory [16], the power coefficients $C_{p 1}$ and $C_{p 2}$ are expressed as a function of tip-speed ratio $\lambda$, blades number $z$ and blade efficiency $E_{b}$ :

$$
C_{p} \cong \frac{16}{27}\left[\exp \left(-0.3538 \cdot \lambda^{-1.2946}\right)-\frac{\lambda}{E_{b}}\right]\left(1-\frac{0.93}{z \sqrt{\lambda^{2}+0.445}}\right)^{2}
$$

By fixing the diameters ratio $D_{i} / D_{e}$ equal to 0.3 , it is possible to size the whole machine. In Table 2 the main design parameters are reported.

Table 2. Main parameters

\begin{tabular}{ccc}
\hline Parameter & Symbol & Value \\
\hline External diameter & $D_{e}$ & $1.08 \mathrm{~m}$ \\
Intermediate diameter & $D_{m}$ & $0.8 \mathrm{~m}$ \\
Internal diameter & $D_{i}$ & $0.32 \mathrm{~m}$ \\
External blade height & $h_{1}$ & $0.14 \mathrm{~m}$ \\
Internal blade height & $h_{2}$ & $0.24 \mathrm{~m}$ \\
Diameters ratio & $D_{i} / D_{e}$ & 0.3 \\
External rotor area & $A_{1}$ & $0.42 \mathrm{~m}^{2}$ \\
Internal rotor area & $A_{2}$ & $0.42 \mathrm{~m}^{2}$ \\
External rotor tip speed ratio & $\lambda_{1}$ & 2.5 \\
Internal rotor tip speed ratio & $\lambda_{2}$ & 1.84 \\
External rotor power coefficient & $C_{p 1}$ & 0.3 \\
Internal rotor power coefficient & $C_{p 2}$ & 0.3 \\
External rotor number of blades & $z_{1}$ & 6 \\
Internal rotor number of blades & $z_{2}$ & 6 \\
rpm & $n$ & 132 \\
Mechanical efficiency & $\eta_{m}$ & 0.9 \\
Electrical efficiency & $\eta_{e}$ & 0.85 \\
\hline
\end{tabular}


From eq. (5), the nominal power calculated for a tidal current velocity peak of $3 \mathrm{~m} / \mathrm{s}$ (Strait of Messina - Italy), will be equal to $2.7 \mathrm{~kW}$, by taking into account an electro-mechanical efficiency of 0.8 .

The CFD analysis of the external rotor is necessary to assess the validity of the blades lift coefficient $\left(C_{L b}\right)$, fixed at 0.4 by means of the zero-dimensional approach [17], it being a key parameter for the blades sizing. Then, it is necessary to evaluate the accuracy of the $C_{p}$ coefficient, estimated by eq. (6).

\section{EXTERNAL ROTOR NUMERICAL ANALYSIS}

This section describes the computational method used to model the machine external rotor and shows some preliminary results of the numerical analysis. The CFD analysis focused on the rotor power coefficient, lift and drag coefficients and efficiency; furthermore, the flow field characteristics and the wake generated behind the rotor are shown.

The numerical simulations are performed for one operating condition considering a free-stream velocity of $3 \mathrm{~m} / \mathrm{s}$ and an angular speed rotor of $132 \mathrm{rpm}$, obtained by considering an optimum tip-speed ratio $\lambda=2.5$, for the external diameter of the external rotor, which is $D_{e}=1.08 \mathrm{~m}$.

\section{Computational and analysis method}

The commercial software FLUENT 15.0 is used to solve the Reynolds Averaged Navier-Stokes (RANS) equations, which are expressed in the following form [18]:

$$
\begin{gathered}
\frac{\partial \rho}{\partial t}+\frac{\partial}{\partial x_{i}}\left(\rho u_{i}\right)=0 \\
\frac{\partial}{\partial t}\left(\rho u_{i}\right)+\frac{\partial}{\partial x_{j}}\left(\rho u_{i} u_{j}\right)=\frac{\partial p}{\partial x_{i}}+\frac{\partial}{\partial x_{j}}\left[\mu\left(\frac{\partial u_{i}}{\partial x_{j}}+\frac{\partial u_{j}}{\partial x_{i}}-\frac{2}{3} \delta_{i j} \frac{\partial u_{l}}{\partial x_{l}}\right)\right]+\frac{\partial}{\partial x_{j}}\left(-\rho \overline{u_{l}^{\prime} u_{\jmath}^{\prime}}\right)
\end{gathered}
$$

where $u_{i}$ denotes the ensemble-averaged velocity component. The additional terms $\left(-\rho \overline{u_{\imath}^{\prime} u_{\jmath}^{\prime}}\right)$ represent the effect of turbulence and are known as Reynolds stresses. Several models are available to close the RANS equations and to compute the Reynolds stresses. The two-equation models are widely adopted and give good accuracy in the simulation of rotating flows [19]. Better results could be obtained by using the Reynolds Stress Model [20], which, for 3D flows, involves more than two equations, or Large Eddy Simulations (LES) and Direct Numerical Simulation (DNS). O'Doherty et al. [20] made a comparison among several turbulence models for the simulation of a horizontal axis tidal turbine. The results show that only small differences occur among all the presented turbulence models. Owing to the lower computation cost and considering that a high degree of accuracy is out of the scope of our work, one of the simplest models and in particular the Realizable k-epsilon model was selected for the current simulations.

The above equations are discretized by a control-volume-based technique. A second order upwind scheme is used for spatial discretization [18]. The convergence criterion for each parameter is set that the residual in the control volume is smaller than $10^{-5}$.

\section{Grids and boundary conditions}

An unstructured domain is built around the foil; it is cylinder shaped with the cylinder axis coinciding with the machine axis (Figure 3 ). In proximity of the foil surface, the grid is refined by creating a prism layer, whose first thickness is $0.01 \mathrm{~mm}$ obtained by imposing the $\mathrm{y}^{+} \sim 1$, according to the following: 


$$
y^{+}=0.172 \frac{\Delta y}{L} R e^{0.9}
$$

where $L$ is the external rotor blade average chord length $(L \sim 0.08 \mathrm{~m})$, and $R e$ is computed considering $L$ as the characteristic length. A growth factor of 1.2 is used. This allows the computation of the hydrodynamic actions in the boundary layer.

A grid dependence study is also presented in order to evaluate the grid independency and to assess the accuracy level of the proposed numerical solution. To this aim, three grids (G1, G2 and G3) were built, with refinement ratio of $\sqrt{2}$ and the lift and drag coefficients are used as key parameters to evaluate the grid convergence.

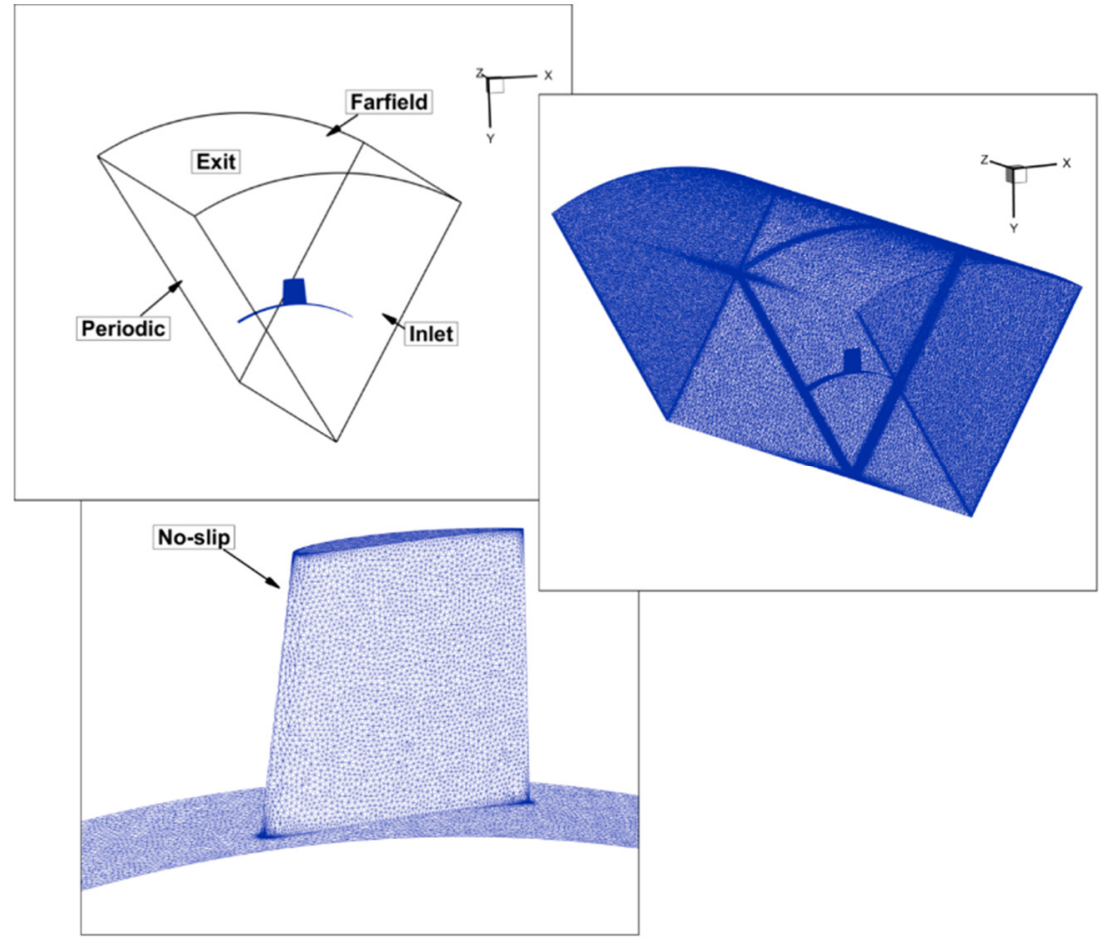

Figure 3. Particulars of the computational domain.

The locations of boundary conditions can be seen in Figure 3; the intake section is located $1 D_{e}$ ahead of the rotor, the outflow section is located $1.5 D_{e}$ behind the rotor and the diameter of the cylindrical domain is $2.5 D_{e}$, where $D_{e}$ indicates the external rotor diameter.

Among the variety of approaches available in FLUENT to model problems involving moving parts, the Single Rotating Reference Frame approach (SRF) is used in the current simulations. This approach, in fact, is suitable for all the cases, which involve a single rotating part (i.e. no stators or baffles): the domain moves with the single rotating frame; the flow in the non-inertial reference frame becomes steady and, consequently, the analysis is simplified and less computationally expensive. When this approach is used, the boundary conditions have to fulfil some requirements: in particular, the no-slip condition on the rotating elements, which are moving walls with the relative frame, is defined by enforcing the fluid relative velocity to zero. In the far-field, which is a non-moving wall with the reference frame, the no-slip condition requires that the absolute velocity is zero on the walls. Finally, a rotationally periodic boundary about the axis of rotation is applied; the computational domain is built for one sixth of the machine with the aim to reduce the computational effort.

The initial and boundary conditions are summarized in Table 3. 
Table 3. Initial and boundary conditions

\begin{tabular}{ccc}
\hline Zone & Boundary type & Initial conditions \\
\hline Inlet & Velocity inlet & $v_{0}=3 \mathrm{~m} / \mathrm{s}$ \\
Exit & Outflow & - \\
Periodic & Periodic & - \\
& Moving wall, & - \\
Foil & relative velocity $=0$ & \\
& Fixed wall, & - \\
Farfield & absolute velocity $=0$ & \\
\hline
\end{tabular}

\section{Grid convergence study}

The three grids G1, G2 and G3 are 1.44, 1.02 and $0.72 \mathrm{M}$ elements, respectively. A comparison, for lift and drag coefficients, among simulation results S1, S2 and S3, which correspond to the fine, medium and coarse grid respectively, is carried out. The results are summarized in Table 4. They show $\varepsilon_{i, i-1}$ as percentage of the finer solution, which is defined as:

$$
\varepsilon_{i, i-1}=\frac{S_{i}-S_{i-1}}{S_{i}} \times 100 \quad i=1 \ldots 3
$$

where $S_{i}$ is the result of finer grid and $S_{i-1}$ is the coarse grid.

Results show that monotonic convergence is reached by increasing the mesh quality, as the difference between the finer and coarser solution decreases with increasing the number of grid points. However, the difference between S1 and S2 is not high enough to justify the use of the finest mesh, and consequently the medium grid G2 is a good compromise between solution accuracy and computational effort.

Table 4. CFD simulations with different grids

\begin{tabular}{cccccc}
\hline \multirow{2}{*}{ Grid } & \multirow{2}{*}{ Grid points } & \multicolumn{2}{c}{$C_{L b}$} & \multicolumn{2}{c}{$C_{D b}$} \\
\cline { 3 - 6 } & & $S$ & $\varepsilon_{12}$ & $S$ & $\varepsilon_{12}$ \\
\hline G1 & $1.44 \mathrm{M}$ & 0.3506 & 0.14 & 0.02790 & 0.53 \\
G2 & $1.02 \mathrm{M}$ & 0.3501 & 0.92 & 0.02805 & 6.34 \\
G3 & $0.72 \mathrm{M}$ & 0.3469 & & 0.02983 & \\
\hline
\end{tabular}

\section{RESULTS}

The validation of the numerical model is made by a comparison of the computed lift and drag coefficients on the blades with the experimental data available in the literature. In particular, the Prandtl correction due to finite foil length, as given by [17]:

$$
a=\frac{a_{0}}{1+\frac{a_{0}}{\pi \cdot A R \cdot e_{w}}}
$$

was applied to the infinite foil lift coefficient, which, for $4^{\circ}$ angle of attack is $C_{L b \text {,inf }}=0.8$; the estimated lift coefficient for the finite length foil, according to eq. (10), is $C_{L b \text {,th }}=0.4$. The simulation value is $C_{L b \text {,sim }}=0.35$. The difference between the estimated and the simulated value amounts to $-12.5 \%$. Similarly, the experimental drag coefficient for the NACA 4412 infinite foil amounts to $C_{D b}=0.006$; the corrected value which considers the finite length effects and, consequently, the lift induced drag is $C_{D b \text {,th }}=0.032$. The drag 
coefficient computed by the numerical simulations is $C_{D b \text {,sim }}=0.028$ with an error amounting to $-12 \%$. These values are in good agreement with the expected theoretical values; furthermore, the path-lines depicted in Figure 4 show that the expected helical wake vortex and the tip vortex on the blade edge are well captured. Consequently, the numerical results can be considered, at this stage of the turbine design process, to be quite useful for a rough estimation of the coefficient of power and of blade efficiency.
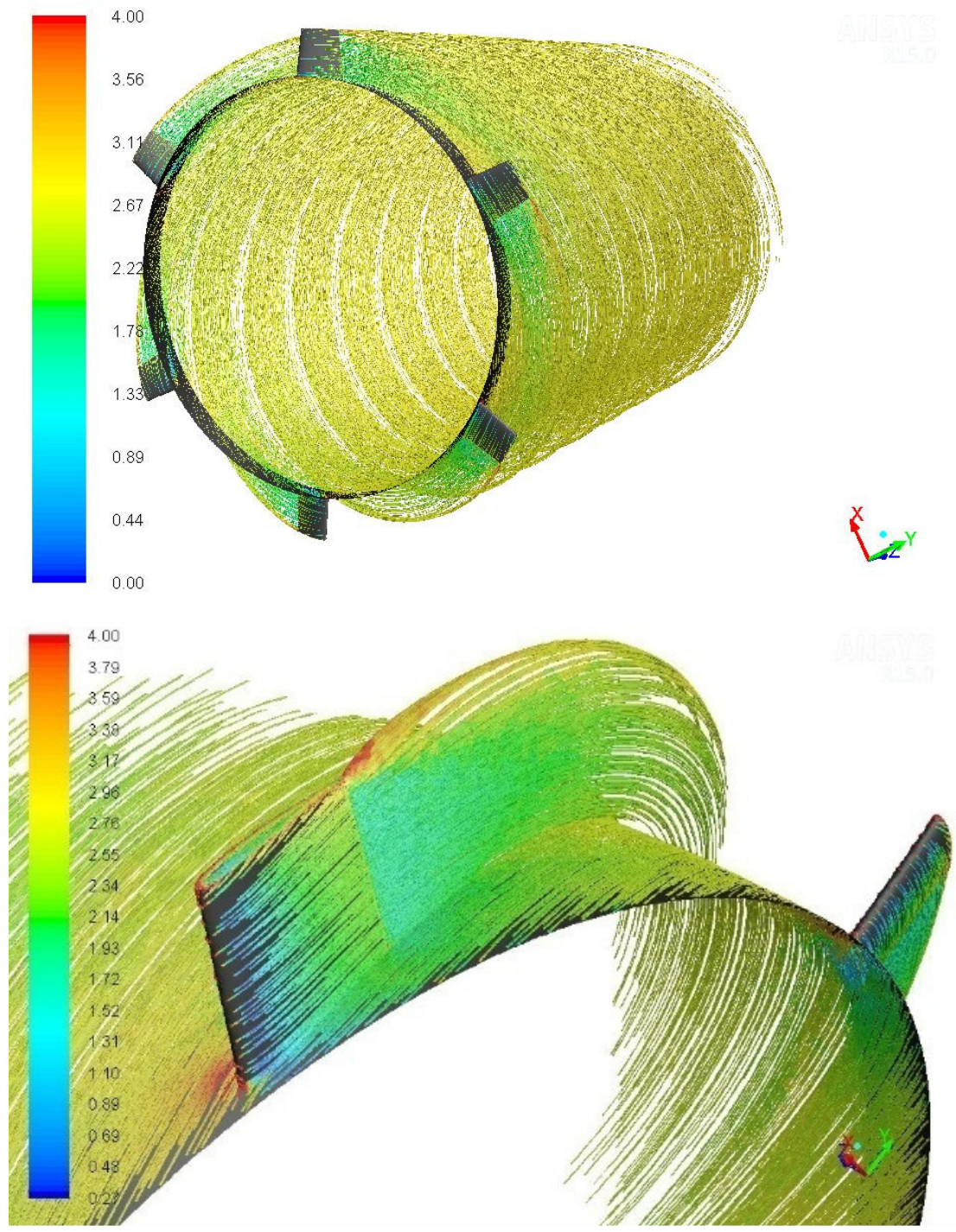

Figure 4. Path lines coloured by velocity magnitude: helical vortex wake region (top); tip vortex (bottom)

The power coefficient is the ratio of the power extracted by the turbine to the total power contained in the free-stream, $C_{p}=P / P_{0}$. The power, $P_{0}$, contained in the free stream and related to the external rotor, is computed as:

$$
P_{0}=\frac{1}{2} \rho \frac{\pi\left(D_{e}^{2}-D_{m}^{2}\right)}{4} v_{0}^{3}
$$

The computed power coefficient is $C_{p \text {,sim }}=0.3$ which is significantly less than the Betz limit (59\%).

The numerical results are also useful for the blade efficiency estimation, which is computed as: 


$$
E_{b}=\frac{C_{L b}}{C_{D b}}
$$

and amounts to $E_{b} \approx 10$.

The prototype under analysis has been designed with 6 blades and $\lambda=2.5$. In these conditions the estimated value of the power coefficient related to the external rotor $C_{p 1}$ [see eq. (6)] is equal to 0.34 . The difference (about $11 \%$ ) is probably due to any effects the one dimensional analysis doesn't consider.

Figure 5 shows the static relative pressure distribution at the inlet and exit of the computational domain; it can be observed that, at the exit, the pressure is lower with respect to the inlet. According to Betz theory for ideal wind turbines, in fact, downstream of the rotor, the power release to the rotor causes both a free stream speed reduction as well as a pressure drop [16]. However, the pressure tends to increase and to reach the inlet value; on the contrary, the stream velocity diminishes and reaches the limit value of $1 / 3$ of its undisturbed value. For real turbines, the blades generate a helical wake vortex, which rotates in the opposite direction with respect to the blades; furthermore, viscous effects must be taken into account. Despite the real phenomena affecting free stream pressure and velocity, their global trend is quite consistent with respect to the ideal theory. This behavior is only slightly visible in the present simulations.
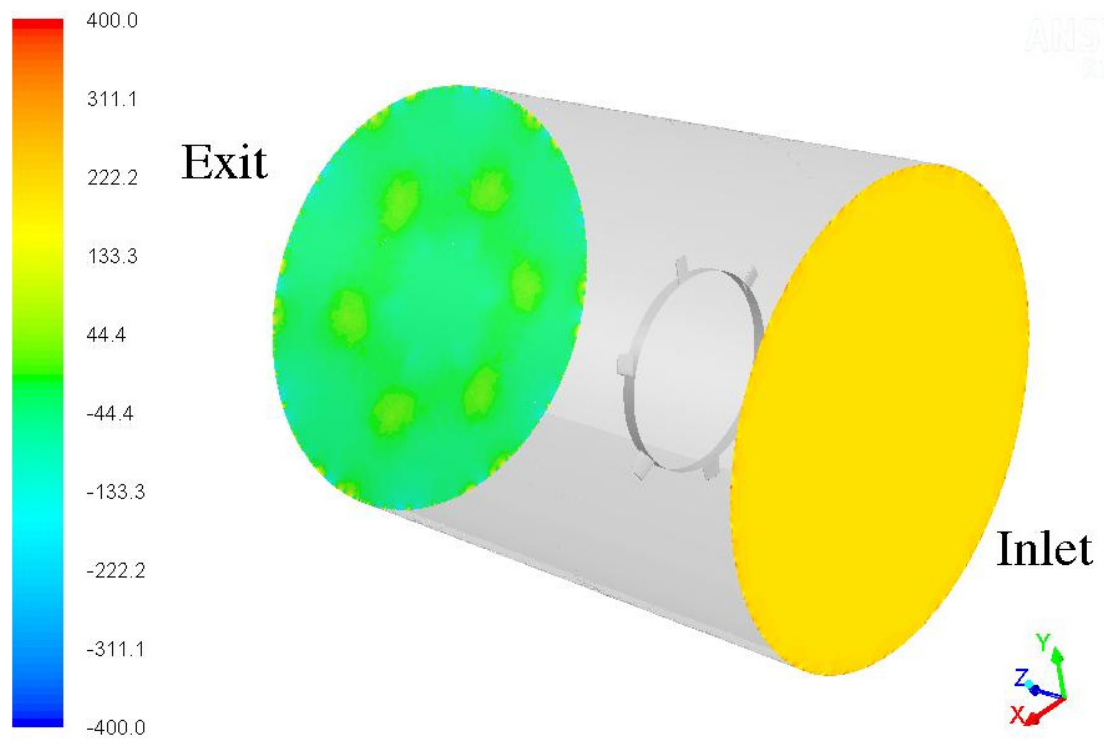

Figure 5. Static pressure distribution at inlet and exit of the computational domain

Figure 6 shows the pressure distribution at several sections of the computational domain along the axis direction on the left, and the corresponding tangential velocity distribution, on the right. The sections are located at $z / D_{e}=0.1,0.5,1$ and 1.5 , where $z=0$ indicates the rotor position and the $z$ direction is positive towards the domain exit. The pressure distribution shows a slow increase from section $z / D_{e}=0.1$, which is behind the rotor, very close to the blades, to $z / D_{e}=1.5$, which is the rotor exit. After the energy release to the rotor blades, the flow undergoes a strong pressure reduction (Figure 6 top-left). The pressure then tends to increase along the axis but it is much lower than the inlet pressure. This suggests that the domain considered for computations is still limited in length and does not allow the expected pressure recovery. The velocity flow field, depicted in Figure 6 (right) shows the tangential velocity distribution and results demonstrate that the pressure increase is due to this velocity component, which reduces in the flow direction. 

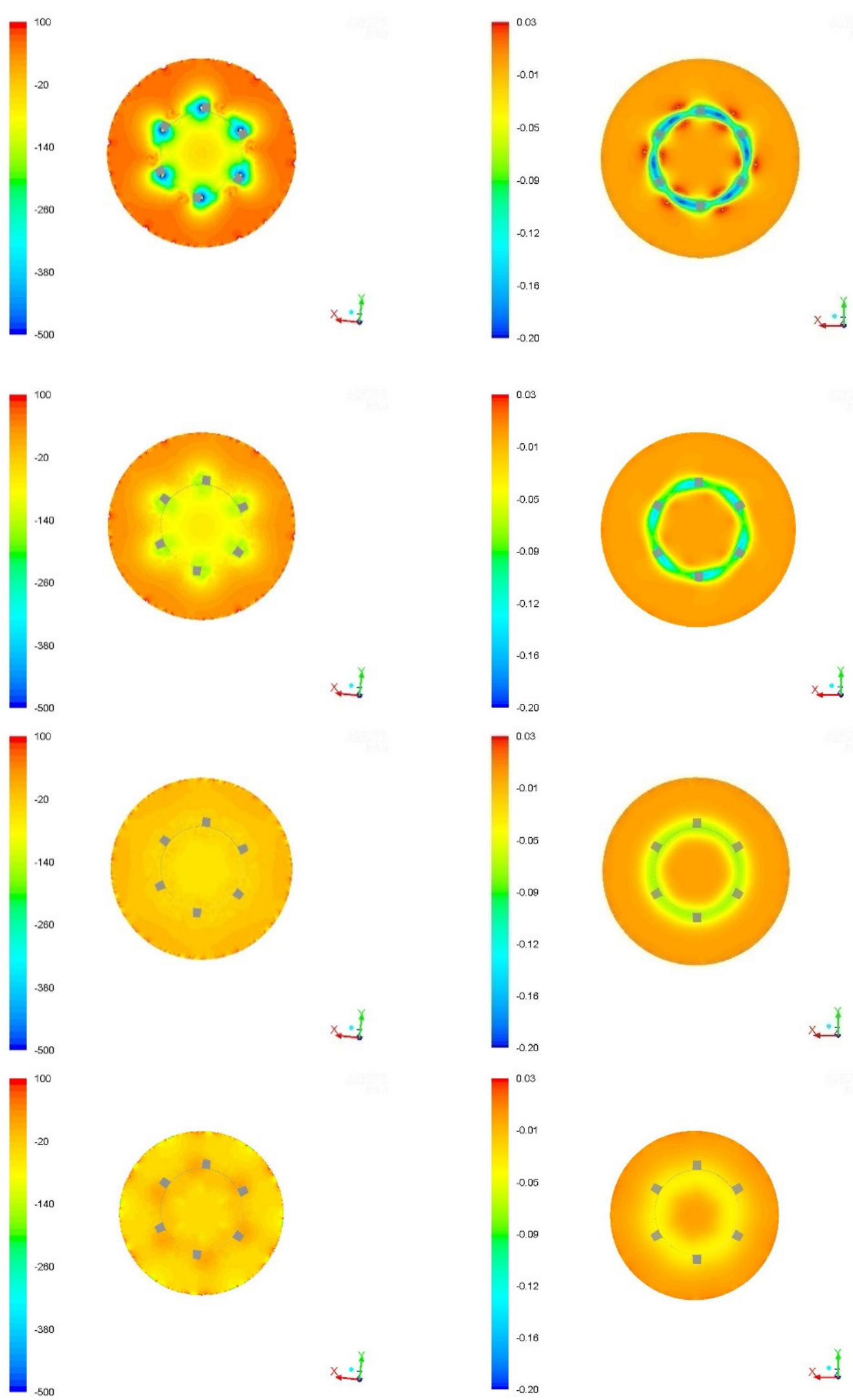

Figure 6. Static pressure distribution (left) and contours of velocity (right) along rotor axis, at sections $z / D=0.1,0.5,1,1.5$

\section{CONCLUSIONS}

The sizing of a turbine kinetic prototype collecting energy from the tidal currents has been done by using a zero dimensional approach. Subsequently, the computational fluid 
dynamic analysis of the flow through external rotor has been carried out with the aim to ascertain the reliability of the zero-dimensional approach.

The results show the lift coefficient on the blade $\left(C_{L b \text {,sim }}=0.35\right)$ is lower than that predicted by zero-dimensional analysis $\left(C_{L b \text {,th }}=0.4\right)$. Moreover, the results show that the rotor power coefficient $\left(C_{p \text {, sim }}=0.3\right)$ is lower than that predicted from the zero dimensional analysis $\left(C_{p}=0.34\right)$.

The predicted maximum power is about $2.7 \mathrm{~kW}$ for tidal currents around $3 \mathrm{~m} / \mathrm{s}$. By taking into account the CFD results, the next steps will optimize the prototype geometry, improving, therefore, the overall performance. Finally, the tests scheduled in an experimental centre in 2017 , will clarify the validity of the proposed method.

\section{HIGHLIGHTS}

- A new marine turbine prototype is designed by means of a zero-dimensional approach;

- The turbine is self-balanced and maintains the equilibrium position by means of a deflector inserted in the stator, a buoy and two counter rotating rotors;

- The blades of the two rotors are designed by means of traditional formulas found in literature;

- Computational fluid dynamic analysisis carried out with the aim to ascertain the reliability of the zero-dimensional approach.

\section{NOMENCLATURE}

\begin{tabular}{ll}
$a_{0}$ & infinite foil lift curve slope \\
$a$ & finite foil lift curve slope \\
$A_{1}$ & external rotor area \\
$A_{2}$ & internal rotor area \\
$A R$ & foil aspect ratio \\
$c$ & chord \\
$C_{D}$ & drag coefficient \\
$C_{D, \text { th }}$ & theoretical value of the drag foil coefficient \\
$C_{D, \text { sim }}$ & simulated value of the drag foil coefficient \\
$C_{L}$ & lift coefficient \\
$C_{L b}$ & blade lift coefficient \\
$C_{L, \text { inf }}$ & infinite lift foil coefficient \\
$C_{L b, \text { th }}$ & blade theoretical value of the lift foil coefficient \\
$C_{L b, \text { sim }}$ & blade simulated value of the lift foil coefficient \\
$C_{p}$ & power coefficient \\
$C_{p 1}$ & external rotor power coefficient \\
$C_{p 2}$ & internal rotor power coefficient \\
$D_{i}$ & internal diameter \\
$D_{e}$ & external diameter \\
$e_{w}$ & Oswald's factor \\
$E_{b}$ & blade efficiency \\
$h_{1}$ & external blade height \\
$h_{2}$ & internal blade height \\
$L_{r}$ & deflector lift \\
$n$ & rotational speed \\
$P$ & power machine \\
$P_{0}$ & flow power \\
$R$ & resultant force \\
$R e$ & Reynolds Number \\
& \\
\hline
\end{tabular}

$[-]$
$[-]$
$\left[\mathrm{m}^{2}\right]$
$\left[\mathrm{m}^{2}\right]$
$[-]$
$[\mathrm{m}]$
$[-]$
$[-]$
$[-]$
$[-]$
$[-]$
$[-]$
$[-]$
$[-]$
$[-]$
$[-]$
$[-]$
$[\mathrm{m}]$
$[\mathrm{m}]$
$[-]$
$[-]$
$[\mathrm{m}]$
$[\mathrm{m}]$
$[-]$
$[\mathrm{rpm}]$
$[\mathrm{kW}]$
$[\mathrm{kW}]$
$[\mathrm{N}]$
$[-]$




$\begin{array}{llc}r & \text { radius } & {[\mathrm{m}]} \\ T_{A} & \text { Archimedes' thrust } & {[\mathrm{N}]} \\ T & \text { blades thrust } & {[\mathrm{N}]} \\ W & \text { machine weight } & {[\mathrm{kg}]} \\ u_{i} & \text { ensemble-averaged fluid velocity component } & {[\mathrm{m} / \mathrm{s}]} \\ v_{0} & \text { undisturbed tidal velocity } & {[\mathrm{m} / \mathrm{s}]} \\ z & \text { number of blades } & {[-]} \\ z_{1} & \text { external rotor number of blades } & {[-]} \\ z 2 & \text { internal rotor number of blades } & {[-]}\end{array}$

\section{Greek letters}

$\begin{array}{llc}\alpha_{p} & \text { incidence angle } & {[\mathrm{rad}]} \\ \beta & \text { positioning angle } & {[\mathrm{rad}]} \\ \beta_{p} & \text { attack angle } & {[\mathrm{rad}]} \\ \eta_{e} & \text { electrical efficiency } & {[-]} \\ \eta_{m} & \text { mechanical efficiency } & {[-]} \\ \phi & \text { functional angle } & {[\mathrm{rad}]} \\ \lambda & \text { tip speed ratio } & {[-]} \\ \mu & \text { radius/tip diameter } & {[-]} \\ \mu & \text { water dynamic viscosity } & {[\mathrm{Pa} \mathrm{s}]} \\ \rho & \text { water density } & {\left[\mathrm{kg} / \mathrm{m}^{3}\right]}\end{array}$

\section{REFERENCES}

1. Nastasi, B. and Basso, G. L., Hydrogen to Link Heat and Electricity in the Transition Towards Future Smart Energy Systems, Energy, Vol. 110, pp 5-22, 2016, https://doi.org/10.1016/j.energy.2016.03.097

2. Esteban, L. and Leary, D., Current Developments and Future Prospects of Offshore Wind and Ocean Energy, Applied Energy, Vol. 90, No. 1, pp 128-136, 2012, https://doi.org/10.1016/j.apenergy.2011.06.011

3. Rourke, F. O., Boyle, F. and Reynolds, A., Tidal Energy, Applied Energy, Vol. 87, No. 2, pp 398-409, 2010, http://dx.doi.org/10.1016/j.apenergy.2009.08.014

4. Manchester, S., Barzegar, B., Swan, L. and Groulx, D., Energy Storage Requirements for In-stream Tidal Generation on a Limited Capacity Electricity Grid, Energy, Vol. 61, pp 283-290, 2013, https://doi.org/10.1016/j.energy.2013.08.036

5. Rourke, F. O., Boyle, F. and Reynolds, A., Marine Current Energy Devices: Current Status and Possible Future Applications in Ireland, Renewable and Sustainable Energy Reviews, Vol. 14, No. 3, pp 1026-1036, 2010, https://doi.org/10.1016/j.rser.2009.11.012

6. Borthwick, A. G. L., Marine Renewable Energy Seascape, Engineering, Vol. 2, No. 1, pp 69-78, 2016, https://doi.org/10.1016/J.ENG.2016.01.011

7. Lo Zupone, G., Amelio, M., Barbarelli, S., Florio, G., Scornaienchi, N. M. and Cutrupi, A., LCOE Evaluation for a Tidal Kinetic Self Balancing Turbine: Case Study and Comparison, Applied Energy, Vol. 185, pp 1292-1302, 2017, https://doi.org/10.1016/j.apenergy.2016.01.015

8. Balluff, S. and Bendfeld, J., Offshore Metocean Station for Energy Purposes, Journal of Sustainable Development of Energy, Water and Environment Systems, Vol. 4, No. 4, pp 333-346, 2016, https://doi.org/10.13044/j.sdewes.2016.04.0026

9. Amelio, M., Barbarelli, S., Florio, G., Scornaienchi, N. M., Cutrupi, A., Minniti, G. and Sanchez Blanco, M., Innovative Tidal Turbine with Central Deflector for the Exploitation of River and Sea Currents in On-shore Installations, Applied Energy, Vol. 97, pp 944-955, 2012, https://doi.org/10.1016/j.apenergy.2011.11.044

10. Barbarelli, S., Amelio, M., Castiglione, T., Florio, G., Scornaienchi, N. M., Cutrupi, A. and Lo Zupone, G., Analysis of the Equilibrium Conditions of a Double Rotor Turbine 
Prototype designed for the Exploitation of the Tidal Currents, Energy Conversion \& Management, Vol. 87, pp 1124-1133, 2014, https://doi.org/10.1016/j.enconman.2014.03.046

11. Barbarelli, S., Amelio, M., Florio, G., Cutrupi, A. and Lo Zupone, G., Transients Analysis of a Tidal Currents Self-Balancing Kinetic Turbine with Floating Stabilizer, Applied Energy, Vol. 160, pp 715-727, 2015, https://doi.org/10.1016/j.apenergy.2015.06.049

12. Barbarelli, S., Florio, G., Amelio, M., Scornaienchi, N. M., Cutrupi, A., Sanchez Blanco, M. and Lo Zupone, G., Engineering Design Study of an Innovative Hydrokinetic Turbine with on Shore Foundation, Journal of Energy and Power Engineering, Vol. 8, No. 3, pp 476-486, 2014.

13. Barbarelli, S., Amelio, M., Florio, G., Scornaienchi, N. M., Lo Zupone, G. and Cutrupi, A., Design Procedure of an Innovative Turbine with Rotors rotating in Opposite Directions for the Exploitation of the Tidal Currents, Energy, Vol. 77, pp 254-264, 2014, https://doi.org/10.1016/j.energy.2014.08.044

14. Barbarelli, S., Amelio, M., Florio, G., Scornaienchi, N. M., Cutrupi, A. and Lo Zupone, G., Transients Analysis of a Tidal Currents Self-balancing Kinetic Turbine with on Shore Basement, Energy Procedia, Vol. 61, pp 962-969, 2014, https://doi.org/10.1016/j.egypro.2014.11.1005

15. Barbarelli, S., Florio, G., Amelio, M., Scornaienchi, N. M., Cutrupi, A. and Lo Zupone, G., Levelized Cost of Energy: A First Evaluation for a Self Balancing Kinetic Turbine, Energy Procedia, Vol. 75, pp 283-293, 2015, https://doi.org/10.1016/j.egypro.2015.07.346

16. Burton, T., Sharpe, D., Jenkins, N. and Bossanyi, E., Wind Energy Handbook (1 ${ }^{\text {st }}$ ed.), Wiley, 2001, https://doi.org/10.1002/0470846062

17. Abbott, I. H. and Von Doenhoff, A. E., Theory of Wing Sections: Including a Summary of Airfoil Data, DOVER Publications, 1959.

18. FLUENT, User's Guide, Ansys.

19. Jo, C. H., Yim, J. Y., Lee, K. H. and Rho, Y. H., Performance of Horizontal Axis Tidal Current Turbine by Blade Configuration, Renewable Energy, Vol. 42, pp 195-206, 2012, https://doi.org/10.1016/j.renene.2011.08.017

20. O'Doherty, T., Manson-Jones, A., O’Doherty, D. M., Byrne, C. B., Owen, I. and Wang, Y. X., Experimental and Computational Analysis of a Model Horizontal Axis Tidal Turbine, Proceedings of the $8^{\text {th }}$ European Wave and Tidal Energy Conference, Uppsala, Sweden, 2009. 